\title{
Response to the Canadian Agency for Drugs and Technologies in Health and Institut national d'excellence en santé et en services sociaux decision regarding nusinersen for Spinal Muscular Atrophy
}

\author{
Craig Campbell, Kathy Selby, Hugh McMillan, Jiri Vajsar, Lawrence Korngut, \\ Bernard Brais, Alex MacKenzie, Maryam Oskoui
}

Keywords: Spinal muscular atrophy, Nusinersen

doi:10.1017/cjn.2018.59

Can J Neurol Sci. 2018; 45: 516-517

As Canadian neuromuscular specialists caring for children and adults with spinal muscular atrophy (SMA), we write to express our disappointment with the recent decision to largely reject access for SMA patients to the anti-sense oligonucleotide drug nusinersen (Spinraza) by Canadian Agency for Drugs and Technologies in Health $(\mathrm{CADTH})^{1}$ and Institut national d'excellence en santé et en services sociaux (INESSS). ${ }^{2}$ After the initial positive decision by Health Canada, and the wide access to nusinersen for a broad range of SMA patients in a number of regions, including the United States and Europe, this decision is very difficult for the SMA community in Canada.

With an estimated incidence of 1 in 11,000 births, SMA is the leading genetic cause of death in infants; without supportive care, children with the most severe form, SMA type I, rarely live to see their second birthday, and those who do, have significant disability. Nusinersen is the first, and currently only, approved treatment for $5 \mathrm{q}$ SMA, with high-level evidence to change the course of the disease. It has the potential to make a difference in the lives of all individuals with SMA types I to III and of all ages. In accordance with this view, after reviewing all available data, Health Canada-in July 2017provided broad approval of nusinersen for all SMA patients. This contrasts with the opinion from Quebec's INESSS, which, while ostensibly recognizing the therapeutic value for type I, asserted that the data from well-designed nusinersen clinical trials ${ }^{3,4}$ are insufficient for SMA types with later age of diagnosis, and the resulting gaps in knowledge are sufficient to not recommend listing the drug for the treatment of all SMA types. On the other hand, the Canadian CADTH recognized the therapeutic value in the most severely affected individuals, but only if started early in their disease course, while rejecting this prescription to all SMA types of varying ages of diagnosis and clinical severity and evolution-in other words, excluding a large part of the SMA community from access to this genetically based treatment. CADTH made their limited approval to type 1 SMA conditional on SMA specialist care, a substantial reduction in price, and the "collection of real-world evidence on the use of nusinersen for the treatment of SMA". Although we view the first two conditions as appropriate, we strongly believe that the third condition is not appropriate; therefore, while the SMA community is actively collecting further data on a broader range of affected individuals, the totality of the data supports broader access than the INESSS and CADTH decisions reflect.

\section{STREngth OF THE DATA}

To fulfill requirements for market approval, nusinersen clinical studies were carefully designed after discussion with the scientific and patient community, as well as regulators both in Canada and around the world. Well-validated primary and secondary end points were designed to assess the clinical impact for infants and children affected by different SMA types for different durations. Type 1 SMA patients younger than 7 months of age and later-onset SMA patients aged 2-12 years were the study populations included in two multi-national, randomized, double-blind, placebo-controlled studies. Importantly, these studies did reach their primary end points showing drug efficacy at interim analysis. ${ }^{3,4}$ The interim results were so compelling that the studies were concluded and children were moved to open-label extension studies. The data from both pre-clinical and clinical studies consistently demonstrate the value

From Department of Pediatrics, Children's Hospital London Health Sciences Centre, Western University, London, Ontario, Canada (CC); Division of Neurology, Department of Pediatrics, BC Children's Hospital, Vancouver, British Columbia, Canada (KS); Division of Neurology, Children's Hospital of Eastern Ontario, Ottawa, Ontario, Canada (HM); Department of Pediatrics, Hospital for Sick Children, University of Toronto, Toronto, Ontario, Canada (JV); Division of Neurology, Department of Clinical Neurosciences, Hotchkiss Brain Institute, University of Calgary, Calgary, Alberta, Canada (LK); Neurogenetics of Motion Laboratory, Montreal Neurological Institute, McGill University, Montreal, Quebec, Canada (BB); Apoptosis Research Centre, CHEO Research Institute, Department of Pediatrics, University of Ottawa, Ottawa, Ontario, Canada (AM); Department of Neurology and Neurosurgery, McGill University, Montreal, Quebec, Canada (MO).

Received February 19, 2018. Final Revisions Submitted April 14, 2018. Date of ACCEPTANCE APRIL 19, 2018.

Correspondence to: C. Campbell, Division of Neurology, Children's Hospital LHSC, B1-177, 800 Commissioners Rd, London, ON, Canada N6A5W9. Email: Craig. campbell@lhsc.on.ca 
of nusinersen with benefits observed in pre-symptomatic infants likely to develop SMA type I or II, and symptomatic patients with SMA type I, II, and III. It is worth noting that children in the control (untreated) groups showed no improvement in motor milestones consistent with the disorder's well-established natural history. The strength of evidence for nusinersen efficacy in SMA type I is sufficiently robust to preclude the ethical use of a placebo arm in future trials. Although high-level evidence has not yet been established for efficacy in individuals with more severe disability and advanced disease, there is the biologically plausible expectation of benefit, and accumulating observational research documenting benefit. Individuals with SMA all share the reasonable goals of halting progression of their disease and maintaining basic functions that are important for independence and quality of life, such as self-care, operating a wheelchair, using a computer and communicating.

The INESSS analysis cites methodological differences between some studies and appears, on this basis, to discount the evidence for nusinersen clinical value. We believe it more appropriate to look at the consistency of demonstrated benefits across the clinical trials notwithstanding study heterogeneity. Indeed, it could be argued that this strengthens the case of efficacy given consistent results using disparate methods. The report also, incorrectly in our view, discounts central positive results and focuses on missing secondary data-for example, the impact of the drug on nutrition, orthopedic and respiratory function in older SMA patients who were not included as participants in the original clinical trials. Finally, to support its claim of insufficient evidence, the INESSS report appears to focus on secondary hierarchical outcome measures that did not reach statistical significance, while ignoring compelling evidence of effect from the same study (e.g. nusinersen impact on upper extremity function). We feel strongly that the totality of the data across studies combined to individual trials and new data should together form the basis of a rapid review of their decision.

\section{Collection of Real-World Evidence}

Finally, we would point out that the real-world evidence collection requested by the agencies is well underway and has attained a level that in our view should lead to approval. The nusinersen clinical program, the largest SMA program to date, comprises multiple studies as part of a long-term commitment to patients to continuously enhance the understanding of nusinersen impact and safety. Biogen, in cooperation with investigators in Canada and abroad, has set up an elaborate program for openlabel study extension, consolidation of SMA registries across the world to collect standardized data, and has launched collaborative research partnerships designed to better assess the "real world" value of nusinersen.

We find ourselves in a discordant situation where Canadian government agencies have approved nusinersen clinical studies, whereas other Canadian agencies point to what they view as critical shortfalls in the design of these same studies. This position undermines public confidence in the regulatory pathways that are ostensibly in place to serve the patient community. Impeding access in Canada to the first effective drug for this devastating disorder in the majority of SMA patients, when there is strong supportive evidence for its efficacy and a clear plan to learn from its broader use, puts into question our scientific approach to evidence-based medicine. Many authorities and payers across the world, having analyzed the same robust clinical data, have approved the prescription of nusinersen across different SMA types, including the United States and seven European countries. In doing so, they are addressing the urgency of a degenerative disease with no alternative therapy. By running counter to the emerging international consensus, the public health systems in Canada are missing this chance to be at the forefront of rare disease treatment, and are neglecting the spirit of care for our fellow citizens that is so dear to all Canadians.

\section{Statement of Authorship}

All authors contributed equally to the creation of this statement.

\section{Disclosures}

$\mathrm{CC}$ reports grants, personal fees and other from Biogen, during the conduct of the study; grants, personal fees and other from PTC Therapeutics; and other from Acceleron, AMO, Biomarin, Catabasis, Pfizer, Sarepta, Wave and BMS, outside the submitted work. KS reports grants from Biogen, grants from IONIS and personal fees from Biogen, during the conduct of the study, and grants from Pfizer, grants from Cytokinetics, grants from PTC Therapeutics, grants from Elly Lilly and grants from Italfarmaco, outside the submitted work.

JV reports grants and personal fees from Ionis and Biogen, during the conduct of the study. LK reports grants and personal fees from Biogen, outside the submitted work. MO reports personal fees from Biogen, grants from Ionis, grants from Biogen, grants from Cytokinetics, grants from Roche and personal fees from Avexis, outside the submitted work. BB, AM and HM has nothing to disclose.

\section{REFERENCES}

1. www.cath.ca. Nusinersen 2018. Available at: https://www.cadth.ca/ nusinersen. Accessed January 18, 2018.

2. www.iness.qc.ca. SPINRAZAMC - Amyotrophie spinale 5q. Dec 2017. Available at https://www.inesss.qc.ca/index.php?id=72\& $\mathrm{L}=1$ \&DemandePluginController\%5Buid\%5D=4426\&DemandePluginController\%5Bonglet $\% 5 \mathrm{D}=4 \&$ DemandePluginController $\% 5$ BbackUrl\%5D=\%252Findex.php\%253Fid\%253D42\%2526no_ cache\%253D1\%2526L\%253D1\&cHash=3d09be911b7a1e0530feb 995d485c2b9. Accessed January 18, 2018.

3. Mercuri E, Darras BT, Chiriboga CA, et al. Nusinersen versus Sham control in later onset spinal muscular atrophy. $\mathrm{N}$ Engl J Med. 2018;378(7):625-35.

4. Finkel RS, Mercuri E, Darras BT, et al. Nusinersen versus Sham control in infantile-onset spinal muscular atrophy. N Engl J Med. 2017;377:1723-32. 